\title{
Gestion du temps verbal et cohésion textuelle en production écrite
}

\author{
Moulay Mohamed TARNAOUI \\ Faculté Polydisciplinaire de Smara - Laboratoire LARLANCO Université IBN Zohr, Agadir - Maroc
}

Reçu le 27 novembre 2019 | Acceptéle 31 décembre 2019

\begin{abstract}
RÉSUMÉ. Notre étude s'inscrit dans une perspective didactique et vise l'étude de certains tiroirs verbaux dans la production écrite des apprenants marocains, particulièrement ceux de la 1ère année du lycée qualifiant, section Sciences Expérimentales. A cet égard, notons que la mauvaise gestion de ces temps ne facilitera pas l'interprétation du texte et rendra en conséquence la tâche du lecteur difficile. En effet, les apprenants passent d'un temps à un autre bien que la consigne soit d'ordre narratif exigeant le respect des temps du récit étudié antérieurement en classe. Substituer un temps verbal à un autre sans tenir compte de la cohésion du texte est une entorse à la norme rédactionnelle. Il découle de cette analyse que la conjugaison, le mode, l'aspect et la concordance des temps continuent à créer de vrais problèmes d'apprentissage chez nos apprenants du FLE en milieu institutionnel.
\end{abstract}

Mots-clés : cohésion textuelle, dysfonctionnements, production écrite, temps verbaux.

\begin{abstract}
Our study is part of a didactic perspective and aims to study some verbal drawers in the written production of Moroccan learners, especially those of the first year of qualifying high school, section Experimental Sciences. In this respect, it should be noted that the mismanagement of these times will not facilitate the interpretation of the text and will make the task of the reader difficult. Indeed, learners go from one time to another although the instruction is of a narrative order requiring the respect of the times of the story studied previously in class. Substituting one verbal time for another without taking into account the cohesion of the text is a departure from the editorial standard. It follows from this analysis that the combination, mode, appearance, and concordance of the times continue to create real learning problems for our FLE learners in institutional settings.
\end{abstract}

Keywords : textual cohesion, dysfunctions, written production, verbal tens.

囷 auteur correspondant : tarnaouimohamed99@gmail.com

Pour citer cet article (Style APA) : Tarnaoui, M.M. (2019). Gestion du temps verbal et cohésion textuelle en production écrite. Francisola: Revue Indonésienne de la langue et la littérature françaises, 4(2), 161-173. doi: 10.17509/francisola.v4i2.24207

\section{INTRODUCTION}

L'un des domaines auxquels la didactique du FLE se réfère pour améliorer l'enseignement / apprentissage, est la linguistique textuelle. Dans les recherches à caractère didactique, on donne de plus en plus d'importance à l'étude des phénomènes textuels en relation avec les différentes compétences qu'un locuteur doit acquérir en langue étrangère. Très souvent, pour interpréter le processus de construction du sens d'un texte, on fait appel aux phénomènes de textualité (Vigier, 2012).

Soulignons dans ce cadre qu'un texte est un ensemble de phrases, mais toute suite de phrases ne forme pas un texte vu qu'entre les phrases d'un même texte, il y a une continuité et le plus souvent une unité de sens (un même thème, un même personnage...). Ainsi, la compétence d'écrire exige la mise en oeuvre d'habiletés et de stratégies que l'apprenant acquiert 
progressivement. Ce dernier doit comprendre que le texte écrit n'est pas une simple succession de phrases, mais une unité complexe (Sabina Mahmudova, 2017) .De même, Il se produirait aussi certainement des ruptures, au bout d'un certain nombre de phrases qui nuisent à la compréhension du message de l'encodeur. A cet égard, certains sous- systèmes de la cohésion textuelle à savoir la ponctuation, l'anaphore et les connecteurs, le temps verbal sont considérés sous un angle particulier qui consiste à compléter l'approche grammaticale par l'approche discursive (Favart et Chanquoy, 2007).

Remontons à Halliday et Hasan (1976) qui affirment que la cohésion concerne l'ensemble des relations sémantiques, identiques pour tous les textes, qui aident à distinguer le texte du non - texte et à découvrir l'interdépendance du contenu de différentes séquences. C'est alors de l'organisation du texte en édifice sémantique qu'il s'agit en ce sens que la cohésion organise les phrases en séquences micro structurelles via les éléments de liages interphrastiques parmi lesquels on peut citer les connecteurs, les anaphores, l'ellipse, la répétition, etc. Pour faciliter l'accès au lecteur, le texte doit se caractériser par une cohésion thématique et sémantique qui maintient une certaine unité au niveau des objets référés et de l'univers représenté.

Vandendorpe (1995, p.9) a considéré six aspects qui génèrent la cohérence du texte, à savoir la continuité thématique, la progression, la cohésion, la gestion adéquate du temps et de l'espace et l'absence d'ellipses trop fortes. Ce chercheur appréhende « la cohésion désigne l'ensemble des opérations qui permettent d'assurer le suivi d'une phrase à une autre. Cette notion se distingue ainsi de la cohérence, qui considère le texte d'un point de vue plus global ».

Dans le domaine de la cohésion qui nous préoccupe davantage, dresser un bilan complet et raisonné des systèmes de marques cohésives d'une langue est difficile comme le remarque Charolles (1995, p.125) :

Lorsque l'on aborde les phénomènes de discours dans cette perspective une des premières difficultés que l'on rencontre consiste à dresser un inventaire raisonné et exhaustif des différents systèmes de marques de cohésion disponibles dans une langue donnée. La réalisation de ce programme va évidemment de pair avec une caractérisation aussi fine que possible des pouvoirs relationnels de ces différents systèmes de marques et de chacune des expressions qui en font partie. Les travaux en la matière ne manquent pas. Dans la période récente, un grand nombre d'études ont en effet paru sur les marques temporelles, l'anaphore, les connecteurs, etc.

Dans la même optique, soulignons deux catégories de rapports : les premiers sont les liens de cohésion, qui joignent les phrases de proche en proche, la première à la deuxième, la deuxième à la troisième, etc., du début à la fin du texte, et expriment ainsi des relations thématiques, logiques, temporelles entre les phrases contiguës d'un texte. Les seconds sont les liens de hiérarchisation, qui indiquent l'importance relative de chaque phrase par rapport aux autres.

Sous le terme de cohésion, Jean-Louis Chiss et Jacques David (2011) décrivent l'ensemble des facteurs qui agissent et le plus souvent interagissent pour que le texte soit non une simple juxtaposition d'énoncés isolés mais une unité discursive autonome liée à un thème donné. Cette unité textuelle doit correspondre à deux nécessités :

- la prise en compte d'une organisation garante d'une interprétation au moins plausible par un auditoire réel ou supposé.

- L'alignement sur des règles de composition ou de mise en texte par l'emploi normatif de marques linguistiques spécifiques.

Les chercheurs et les enseignants se rappellent de l'apport de Benveniste (1966) et de Weinreich (1973) en matière de temporalité mais autres recherches actuelles ont traité la problématique du temps verbal en abordant d'autres paliers de l'analyse. Les contributions sont nombreuses et témoignent de la richesse et de la dynamique du champ. Sur ce plan, comme nous ne pouvons pas les citer toutes dans le cadre de la revue de littérature, nous citons quelques études les 
plus proches de notre thématique. A titre d'exemple, Sophie Azzopardi et Jacques Bres (2011) ont mis l'accent sur le temps verbal et énonciation. Ils ont essayé de montrer comment la catégorie de l'énonciation, approfondie par celle de dialogisme, permet d'expliquer les parallélismes comme les divergences observables dans les emplois du futur et du conditionnel en français. Ces chercheurs ont proposé une hypothèse concernant la valeur en langue de chacun d'eux : le futur est un «ultérieur du PRÉSENT»; le conditionnel, un « ultérieur du PASSÉ ».

De son côté, Mona Al wohaib (2012) voit que la langue arabe considère les aspects et les temps sous un angle très spécifique. Beaucoup d'étudiants ont bien du mal à traduire et comprendre ces phénomènes, notamment dans les cas où la langue arabe est la langue seconde. Cette difficulté a été occultée, c'est pour cela elle a cherché à en décrire les fonctionnements temporels et aspectuels de façon à ce que les apprenants puissent se familiariser sans difficulté avec les structures linguistiques. Le temps est l'une des composantes les plus importantes de l'action et permet l'analyse de l'événement porté par le verbe. De ce fait, le temps que construisent la morphologie verbale et la temporalité deviennent indissociables.

Dans le même sillage, Catherine Fuchs, Pierre Le Goffic et Mathieu Maillard (2014) ont étudié l'emploi des temps dans L'Occupation des sols. À travers des représentations d'événements et de situations, les temps verbaux contribuent, dans un récit classique à la cohérence du récit. Dans le texte d'Echenoz, au contraire, coupes discontinues, ruptures de plans, raccourcis, avancées et retours en arrière donnent, pour ainsi dire, une vision stroboscopique de l'histoire, laissant au lecteur le soin de combler les "trous » du récit et l'obligeant à tisser lui-même la trame narrative, afin d'en restituer la cohérence globale.

L'objectif primordial de notre recherche est d'étudier le temps verbal déviant dans la production écrite de nos scripteurs du FLE dans le dessein de vérifier si ce sous- système de la cohésion du texte utilisé par nos lycéens contribue à rendre leur écrit clair et lisible ou au contraire favorise l'opacité pour le lecteur. Il serait crucial de suggérer quelques pistes didactiques en vue de remédier à cette situation pédagogique qui caractérise l'enseignement $d u$ FLE en milieu institutionnel marocain.

Dans cette perspective, il est indispensable de poser les questions suivantes: les lycéens marocains du FLE utilisent-ils adéquatement le temps verbal dans le cadre d'une séquence narrative? Quels aspects de la temporalité bloquent l'apprentissage du FLE au lycée qualifiant (temps, le mode, l'aspect, etc. ?Quels temps posent réellement problème aux apprenants? La mauvaise exploitation des tiroirs verbaux ne génère-elle un problème de lecture, d'interprétation?

Pour accomplir cette tâche, nous avons consciemment limité notre recueil aux phénomènes $d^{\prime}$ 'organisation textuelle les plus saillants concernant le temps verbal. Nous avons focalisé notre attention sur les différentes substitutions des temps verbaux employés par nos apprenants, sujets de l'enquête. C'est pour cela, nous avons ainsi retenu les faits qui sont déficients qui inhibent la cohésion du texte. Celle-ci est appréhendée comme l'ensemble des facteurs qui agissent pour que le texte soit, non la simple juxtaposition d'énoncés isolés, mais une unité discursive autonome liée à un thème donné.

\section{MÉTHODE}

\subsection{Collecte de données et procédure d'analyse :}

Le but que nous entreprenons est l'étude synchronique d'un sous- système de la cohésion textuelle $\mathrm{du}$ groupe expérimental, Tronc Commun Sciences expérimentales dans l'intention de vérifier les déviations relatives aux temps verbaux qui affectent la production et par conséquent altèrent la compréhension du message. Dans cette perspective, le recours aux données du terrain s'avère essentiel en vue d'optimaliser l'apprentissage. 
A cet égard, les activités de production écrite ont pour objectif de faire acquérir à l'élève une véritable compétence rédactionnelle, c'est-à-dire de l'amener à utiliser l'écrit de manière autonome soit en tant que moyen de communication répondant à une visée spécifique, soit comme forme d'expression personnelle. Nous avons choisi ainsi cette technique comme source de production de nos données parce qu'elle permet d'obtenir des corpus ouverts où les apprenants ne se sentent pas limités par des contraintes sévères et inhibitrices.

Quant à l'approche investie dans le cadre de cette investigation, elle demeure textuelle. Rappelons qu'elle est fondée sur le " texte ", c'est le point de départ de l'enseignement de toutes les activités pédagogiques. Elle constitue une composante des pratiques éducatives que les sciences pédagogiques modernes utilisent pour l'installation des compétences nécessaires. Ainsi, un changement important est survenu dans le processus d'enseignement/ apprentissage: l'apprenant n'est plus un simple récepteur et consommateur de connaissances, il devient un acteur qui interagit avec elles. On est passé ainsi de l'enseignement vers l'apprentissage productif. Quant à l'enseignant, il devient un animateur, un conseiller, un guide, un gestionnaire des situations d'apprentissage .L'approche textuelle représente un levier important dont se sert l'enseignant afin de stimuler les capacités de l'apprenant et de les développer. Elle donne, en outre, la possibilité à l'apprenant d'exploiter ses capacités en s'appuyant sur ses prérequis.

Notre procédure consiste à collecter les différents temps utilisés de façon déficiente par notre groupe expérimental et les répertorier selon les tiroirs verbaux puis examiner ensuite les différentes substitutions erronées auxquelles les apprenants recourent pour exprimer leurs idées et leurs conceptions .Cette méthode vise l'analyse des anomalies de la temporalité et la recherche des solutions didactiques pour l'optimisation de l'apprentissage du FLE. A cet effet, nous avons formulé le sujet pour les TCS de la manière suivante :
Sujet: Imaginez une suite et une fin au récit suivant : Il était une fois un jeune homme qui pêchait au bord de la mer... Une nuit,.

\subsection{Participants}

Notre choix s'est porté sur la rédaction d'un texte narratif. Cette dernière aide les lycéens à être à l'aise dans l'expression de leurs idées et libres d'exploiter toutes les ressources de leur compétence linguistique. Il est donc question d'une classe de 43 élèves, filière sciences expérimentales appartenant au lycée Faissal Ben Abdelaziz, direction provinciale Inezgane-Ait Melloul. Ce groupe a été choisi fortuitement. Ce prélèvement est représentatif de l'ensemble des lycéens qui sont exposés à une approche par les compétences en FLE. Soulignons que les classes sont presque similaires puisque la distribution dépend du logiciel Massar. De même leur niveau linguistique et culturel est identique. L'épreuve que les lycéens ont passée a lieu le 20 octobre 2016. L'âge des apprenants varie entre 15 et 16 et sont originaires de l'Académie Souss- Mass.

Le corpus sur lequel nous avons travaillé les déviations de la temporalité était consacré en principe aux déviations anaphoriques dans le cadre d'une recherche doctorale intitulée : «Cohésion textuelle dans la production écrite : déviations anaphoriques et stratégies didactiques » (Moulay Mohamed Tarnaoui, 2019). Or l'anaphore ne constitue qu'une composante de la cohésion. En effet, nous n'avons pas analysé les connecteurs, la ponctuation, les aspects de la temporalité, les constructions détachées, le découpage en paragraphe, etc. qui causent de véritables problèmes rédactionnels chez nos lycéens et par conséquent favorisent l'émergence de déviations textuelles. Ainsi, il serait fondamental, à notre sens, d'entreprendre des études futures sur les questions de la cohésion textuelle en relation avec les activités de production écrite. La contribution actuelle s'inscrit de facto dans cette optique. 


\section{RÉSULTATS ET DISCUSSION}

Nous avons présenté dans un premier temps l'inventaire des tiroirs verbaux localisés dans la production narrative de notre groupe expérimental; il est donc question de la répartition des temps dans les récits du TCS. Dans un second temps, nous attaquons particulièrement les substitutions déficientes de ces tiroirs verbaux qui créent des problèmes de compréhension et d'interprétation.

Tableau 1. Répartition des temps dans les récits du TCS :

\begin{tabular}{|c|c|c|c|c|c|c|c|}
\hline $\begin{array}{c}\text { Copies/ } \\
\text { temps }\end{array}$ & $\begin{array}{c}\text { Passé } \\
\text { simple }\end{array}$ & Imparfait & p. composé. & prés. & $\begin{array}{l}\text { p. } \\
\text { ant. }\end{array}$ & p. q. parfait. & inf. \\
\hline 1 & 7 & 14 & 8 & 9 & 0 & 0 & 5 \\
\hline 2 & 3 & 11 & 4 & 8 & 0 & 0 & 4 \\
\hline 3 & 4 & 3 & 3 & 15 & 0 & 0 & 2 \\
\hline 4 & 5 & 14 & 2 & 8 & 0 & 0 & 1 \\
\hline 5 & 6 & 14 & 0 & 6 & 0 & 0 & 3 \\
\hline 6 & 5 & 8 & 4 & 14 & 0 & 0 & 0 \\
\hline 7 & 0 & 11 & 1 & 12 & 0 & 0 & 6 \\
\hline 8 & 0 & 1 & 2 & 9 & 0 & 0 & 7 \\
\hline 9 & 6 & 2 & 1 & 8 & 0 & 0 & 0 \\
\hline 10 & 0 & 17 & 6 & 11 & 0 & 0 & 1 \\
\hline 11 & 3 & 21 & 23 & 19 & 0 & 0 & 0 \\
\hline 12 & 2 & 9 & 12 & 10 & 0 & 0 & 4 \\
\hline 13 & 5 & 11 & 1 & 15 & 0 & 0 & 1 \\
\hline 14 & 8 & 7 & 2 & 8 & 0 & 0 & 3 \\
\hline 15 & 10 & 17 & 9 & 10 & 0 & 0 & 0 \\
\hline 16 & 9 & 8 & 2 & 18 & 0 & 0 & 1 \\
\hline 17 & 1 & 11 & 3 & 22 & 0 & 0 & 4 \\
\hline 18 & 2 & 9 & 2 & 34 & 0 & 0 & 2 \\
\hline 19 & 1 & 6 & 0 & 22 & 0 & 0 & 0 \\
\hline 20 & 5 & 11 & 3 & 16 & 0 & 0 & 1 \\
\hline 21 & 10 & 10 & 0 & 15 & 0 & 0 & 2 \\
\hline 22 & 5 & 20 & 1 & 8 & 0 & 0 & 5 \\
\hline 23 & 12 & 19 & 2 & 10 & 0 & 0 & 0 \\
\hline 24 & 9 & 14 & 0 & 6 & 0 & 0 & 6 \\
\hline 25 & 8 & 31 & 0 & 9 & 0 & 0 & 5 \\
\hline 26 & 7 & 8 & 5 & 23 & 0 & 0 & 11 \\
\hline 27 & 1 & 12 & 1 & 11 & 0 & 0 & 0 \\
\hline 28 & 10 & 18 & 6 & 11 & 0 & 0 & 1 \\
\hline 29 & 0 & 17 & 20 & 10 & 0 & 0 & 0 \\
\hline 30 & 4 & 14 & 8 & 15 & 0 & 0 & 4 \\
\hline 31 & 6 & 11 & 4 & 9 & 0 & 0 & 2 \\
\hline 32 & 8 & 6 & 3 & 8 & 0 & 0 & 8 \\
\hline 33 & 5 & 4 & 2 & 9 & 0 & 0 & 3 \\
\hline 34 & 3 & 15 & 0 & 7 & 0 & 0 & 3 \\
\hline 35 & 6 & 18 & 4 & 6 & 0 & 0 & 1 \\
\hline 36 & 5 & 11 & 1 & 2 & 0 & 0 & 4 \\
\hline 37 & 8 & 7 & 2 & 8 & 0 & 0 & 5 \\
\hline
\end{tabular}




\begin{tabular}{cccccccc}
\hline $\begin{array}{c}\text { Copies/ } \\
\text { temps }\end{array}$ & $\begin{array}{c}\text { Passé } \\
\text { simple }\end{array}$ & Imparfait & p. composé. & prés. & $\begin{array}{c}\text { p. } \\
\text { ant. }\end{array}$ & p. q. parfait. & inf. \\
\hline 38 & 6 & 12 & 1 & 6 & 0 & 0 & 3 \\
39 & 10 & 7 & 6 & 11 & 0 & 0 & 1 \\
40 & 3 & 10 & 23 & 7 & 0 & 0 & 7 \\
41 & 12 & 30 & 12 & 6 & 0 & 0 & 7 \\
42 & 0 & 11 & 1 & 7 & 0 & 0 & 2 \\
43 & 4 & 8 & 2 & 9 & 0 & 0 & 6 \\
\hline
\end{tabular}

Le tableau suivant a pour objectif de dresser un comptage complet des principaux temps utilisés par le groupe-classe TCS et met l'accent sur les temps les plus utilisés par les lycéens dans une séquence narrative. Cependant, dans les copies du même corpus, l'usage des temps est-il cohérent et adéquat?

L'inventaire vise en premier lieu la répartition des tiroirs verbaux dans les copies des lycéens du TCS. Il est question de collecter les différents temps utilisés par nos apprenants et les répertorier selon les tiroirs verbaux puis examiner ensuite les différentes substitutions déficientes auxquelles les apprenants recourent pour exprimer leurs idées et leurs conceptions dans un texte narratif.

A vrai dire, ces derniers trouvent des difficultés d'apprentissage dans le maniement des temps verbaux. L'un des tiroirs qui demeure problématique est le passé simple(P.S). En effet, 4 copies parmi 43 (Copie 5, Copie 15 Copie 28 et Copie 41) où figure le passé simple et utilisé à bon escient, soit 9./. Là nous émettons l'hypothèse du remplacement du P.S. par un autre temps. C'est un tiroir qui crée des difficultés à l'apprenant en raison de sa conjugaison et de sa valeur. De surcroit, la consigne est de nature narrative $\mathrm{du}$ moment que nous demandons aux apprenants de rédiger la suite d'un récit. La récurrence des temps du récit est ainsi une donnée incontournable de l'histoire racontée par les apprenants; d'où le passé simple, l'imparfait, le plus que parfait et le présent de narration.

Ensuite, nous avons remarqué à partir du relevé que nos apprenants ne font pas appel au passé antérieur et au plus que parfait. En effet, nous n'avons repéré aucune trace de ces deux tiroirs verbaux. Ce qui nous incité à réfléchir davantage sur les aspects de la temporalité : ces deux temps sont enseignés mais ils ne sont exploités par les lycéens à l'écrit et à l'oral et donc ils restent dans l'ombre. Autrement dit, il y a un hiatus entre la relation d'enseignement et la relation d'apprentissage. Si les deux temps étaient appris réellement, ils devraient être exploités. A cet effet, à part le passé composé, les autres temps composés ne sont pas investis. Ce dernier est justifié par le fait qu'au collège, c'est le temps le plus utilisé même aux examens normalisés. En outre, c'est le temps de l'oral.

En troisième lieu, les apprenants recourent plus à l'imparfait, au passé composé et au présent qu'aux autres temps. Notons que ce recours demeure déficitaire en raison du temps, du mode ou de l'aspect adéquat qu'il faut utiliser. Quant à l'infinitif exprimé par l'apprenant, il devient un joker puisqu'il transgresse la norme de l'infinitif. Dans ce genre de situation, seul l'environnement linguistique des verbes en question nous permet de « deviner » le temps que l'apprenant désire employer en nous fiant surtout au temps auquel les verbes placés avant ou après l'infinitif sont exprimés.

En quatrième lieu, les apprenants gèrent maladroitement le système verbal $\mathrm{du}$ fait qu'ils font entorse à la norme de la tripartition passé/présent/futur. De plus, les substitutions relevées dans les productions écrites sont déviantes et manifestent la compétence transitoire de l'apprenant.

Notons que le principe essentiel que tout enseignant de français langue étrangère devrait méthodiquement observer est que l'écriture est un acte qui s'apprend. Il s'agit d'une pédagogie heuristique. De ce fait, la première stratégie que l'on doit concevoir consiste à observer notre action didactique 
sous forme de projet pédagogique qui consiste à prendre les lycéens tels qu'ils sont et non tels qu'ils sont censés être.

\subsection{Substitutions déviantes au niveau de la temporalité \\ Les substituions traitées sont} considérées déviantes du point de vue de la norme du moment que le tiroir verbal exigé est remplacé par un autre. C'est une déviation temporelle. Nous allons consacrer cette partie au relevé du mode indicatif.

\subsubsection{Substitution du présent à l'imparfait}

Dans les énoncés ci-dessous, nous citons certaines occurrences de maladresse au niveau de la temporalité. A cet effet, les apprenants ont substitué le présent à l'imparfait bien que les valeurs et les temps des deux tiroirs verbaux soient divergents :

- Un jour le jeune homme se promène. Soudain rencontre un homme (Copie1).

- Il était une fois un jeune homme qui pêchait au bord de la mer.il vendait ce qu'il prenait et le reste, il le mangeait. Une nuit, il rêve $d^{\prime}$ 'une boit d'or et un nouveau pays plein de fleurs(Copie1).

- Il a essayé à la dernière fois tire la sirène doré. Le pécheur a peur, la sirène doré avec le pêcheur (Copie2)

- Il a trouvé sa femme entrain de dormir, il est habié de vêtements de roi, il est dans un château(Copie2)

- Soudain, il voyait un petit garçon qui est assis à l'angle de cette grotte et pleure. Ce garçon porte des vêtements qu'il y a des sangs(C.4).

- pendant quelques heures, il regarde au bord de la mer, il vois un petit garçon dans la mer, il mort. Quand l'enfant est sorti, il entre dans une boite(C.11).

- Une nuit ce pêcheur va pour pêcher quelques poissons. Soudain, le climat est changé, la rafale a commencé (C.31).
- Il était une fois un jeune homme qui pêchait au bord de la mer.il vendait ce qu'il prenait et le reste, il le mangeait. Une nuit, Il partire à le bord de la mer comme d'habitude mais il ne pêche pas. Il fait des tentative mais aucune résultat(Copie2).

- Le pécheur rentre chez lui mais est surpris par ce que la barque a changé en palais(Copie2).

- Enfin le pécheur et la femme il vive dans une joie totale (Copie2).

- Il était une fois un jeune homme qui pêchait au bord de la mer.il vendait ce qu'il prenait et le reste, il le mangeait. Une nuit, le pêcheur doit porter tous ce qu'ils pêche à sa maison. Soudain, le pêcheur était très peur pour ce nuit car les voleurs vont voler ses poissons (Copie 3).

- Il était une fois un jeune homme qui pêchait au bord de la mer.il vendait ce qu'il prenait et le reste, il le mangeait. Une nuit, dans la maison, il mange le diner avec sa femme dans la salle à manger et ils parte pour dormir. Le jeune homme sent qu'une chose va arriver.(C.5)

- La nuit suivante le jeune homme se réveille et lave son visage et mange son petit déjeuner et ramasse ces affaires de pêche et va à la mer. Quand le panier était plein, il va au marché de poisson.(C.5)

- Quand il vendait le poisson, il a parti à la maison manger le déjeuner il revient le premier à la mer pour pêchait encore beaucoup de poissons. Il prennent sa canne et commence à pêcher.(C.5)

- Comme d'habitude il vendut les poissons que va pêcher et il doit porter ce qui reste pour le manger... il connait qu'il va devenir tres rich et il va vivre dans un grand palais(C.8). 
- le pêcheur ne savait pas ce qu'il va dire et ce qu'il va faire(C.10).

- quand il a lancé la canne il ne peut pas la tirer mais la surprise était une boite(C.12).

- une nuit, le jeune homme a lancé sa canne. Après deux heures, quelque chose fait tirer l'hameçon. Le jeune homme a peur mais rien du tout. Il voulait savoir ce qui ce passe sous l'eau (C.17)

- il observe un grand poisson qui nage à l'intérieur de la mer, il va vite à son bateau et prende son filet et sa canne de pêche(C.39).

- en retournant vers sa cabane qui est pas loin du littoral, il a rencontré un homme il a le même âge que lui et il demande l'hospitalité pour quelques jours parce qu'il a aucune place où il peut vivre(C.41).

- Le jour suivant le jeune homme va pour pêcher. Il lance sa canne. Soudin, il tire de tous ses forces un sirène. Le jeune homme est surpris et perd sa connaissance (C.43).

- Soudain, dans sa chambre, il trouve une femme qui pleure tout seul, son visage a complétement attiré le pêcheur. Et il l'a questionné de ce qui a provoqué sa tristesse(C.43).

Comme nous l'avons constaté supra, les apprenants ont substitué le présent à l'imparfait. Cette substitution demeure déviante vu que les présents localisés dans le corpus doivent être à l'imparfait. Il faut souligner que nous n'avons pas répertorié aucun verbe au présent de narration. En outre, les lycéens en question ne maîtrisent pas la notion du temps, de l'aspect et du mode. L'examen de l'emploi des tiroirs verbaux dans les énoncés ci-dessus nous permet de dire que la substitution du présent à l'imparfait est due à la méconnaissance des règles de concordance et à l'ignorance de temps.

Concernant la concordance des temps, un apprenant arabophone trouve des difficultés devant l'apprentissage du système temporel du français car, outre sa complexité propre, il diffère totalement de celui de l'arabe. Les temps et les modes en français servent à traduire des nuances qui dépassent souvent la simple indication temporelle à laquelle se limite le système verbal de l'arabe avec ses deux catégories d'accompli et d'inaccompli. En d'autres termes, en arabe, il n'existe pas une forme spécifique correspondant à l'imparfait en français. Celui-ci est rendu par la forme de l'inaccompli(le présent) qui exprime l'aspect ; le temps est exprimé par le contexte.

\subsubsection{Substitution du passé composé à l'imparfait}

Un cas constaté chez nos apprenants du lycée qualifiant est le recours au passé composé au dépend de l'imparfait tant attendu dans des séquences narratives. C'est un écart par rapport à la norme. De plus, la consigne oblige l'usage des temps du récit. Les exemples suivants en témoignent :

- Il a essayé à la dernière fois tire la sirène doré. Le pécheur a peur, la sirène doré est parlé avec le pêcheur (Copie2)

- Il a trouvé sa femme dormir depuis des heures, il est habié de vêtements de roi il est dans un chateau(Copie2).

- en retournant vers sa cabane qui est pas loin du littoral, il a rencontré un homme il a le même âge que lui et il demande l'hospitalité pour quelques jours parce qu'il a aucune place où il peut vivre(C.41).

- La sirène a accepté. Le jeune homme a été heureux et a demandé la sirène au mariage(C.43)

- Après trois mois, le jeune homme a trouvé la princesse. Il a commencé les coutumes du mariage (C. 29).

- Le pécheur est choqué. Il a caché la boite dans une grotte. Ce pécheur est devenu un grand marchand dans l'île. Il a acheté un 
grand bateau et toute sa famille a été contente(C.30).

- le jour suivant, ce jeune homme est parti à la mer et a lancé le filet... (C.36).

- après des heures, le magicien de cette rivière a trouvé le pêcheur avec le renard. Il a transformé le pêcheur en poisson(C.26).

- Il se peut que les élèves soient habitués au passé composé durant la période du collège et ils ne parviennent pas encore à intégrer les temps du récit dans leur production.

Les occurrences produites par le groupe expérimental sont déviantes dans la mesure où le tiroir convenable est l'imparfait. En effet, il s'agit d'actions qui durent dans le passé. L'apprenant est contraint d'investir l'indicatif imparfait au lieu du passé composé pour véhiculer l'aspect duratif dans le passé. Nous déduisons que la notion de l'aspect demeure problématique dans l'enseignement/apprentissage du FLE.

\subsubsection{Substitution du présent/ PC au passé simple \\ Les occurrences suivantes révèlent que} les substitutions produites par les lycéens du TCS sont incohérentes. En effet, nos lycéens font appel au présent ou bien au passé composé là où le lecteur attend le passé simple. Comme signalé dans le tableau, ce tiroir verbal constitue un obstacle d'apprentissage pour les apprenants. Dans le dessein de détourner la difficulté de ce temps verbal, le présent et le passé composé deviennent des procédés ou des stratégies compensatoires :

- Soudain, il voit un boit plein d'or. Il va découvrir la boit. Il trouve la boit plein d'or. Le pêcheur est heureux (Copie1).

- Un jour le jeune homme se promène. Soudain rencontre un homme (Copie1).

- Le pécheur rentre chez lui mais vite il est surpris par ce que la barque a changé en palais(Copie2).
- Soudain, il a trouvé une grande boite au bord de la mer, il s'approche de la boite et une sirène sort(Copie3).

- Le jeune homme ne pêchait rien et après deux heures d'attente, le filet est bougé...Ensuite, le jeune est bouleversé car la sirène était magnifique(C.10)

- Lorsqu'il emmenait cette boite magique à la maison, sa famille est choqué.

- Soudain, les vagues fortes ont commencé une tempête et le pêcheur a peur de cela et d'un grand vague, il sort un diable. Il vient avec une boite d'or...(C.38).

- Le jour suivant le jeune homme va pour pêcher. Il lance sa canne. Soudin, il tire de tous ses forces un sirène. Le jeune homme est surpris et perd sa connaissance (C.43).

- Une nuit ce pêcheur va pour pêcher quelques poissons. Soudain, le climat est changé, la rafale a commencé (C.31).

- Soudain, le pêcheur voit un diable, il est inquiète et peur(C.36).

- Soudain, il voit une belle fille au bord de la mer mais il ne sait pas que c'est une sirène...(C.28).

- Soudain, dans sa chambre, il trouve une femme qui pleure tout seul, son visage a complétement attiré le pêcheur. Et il l'a questionné de ce qui a provoqué sa tristesse(C.43).

Considérons les exemples ci-dessus, les apprenants ont enfreint la norme de la temporalité du fait qu'ils ont remplacé le P.S. soit par le présent ou le P.C. Cette déviation est expliquée par la non maîtrise de la conjugaison du passé simple qui exige de l'apprenant et de l'enseignant des efforts. Ensuite, l'apprenant ignore l'emploi effectif de l'aspect: quand faut-il recourir au passé simple? A cela s'ajoute que les lycéens qui sont soumis à l'enquête n'exploitent pas les marqueurs temporels tels: soudain, tout à coup, subitement, brusquement, une nuit, etc. qui entraînent le P.S. puisque les actions 
sont soudaines, le récit est d'ordre singulatif où l'on raconte une fois ce qui s'est passé une fois.

\subsubsection{Substitution de l'imparfait au passé simple et vice versa \\ Ce sont les deux temps du récit les} plus utilisés. A ce propos, Bronckart (1985: 87) écrit: «La discriminativité par rapport aux autres catégories de textes est assurée avant tout par le sous- système temporel passé simple - imparfait, principale trace saillante et récurrente du repérage narratif ». Les énoncés ci-dessous illustrent la confusion entre les deux temps. La mauvaise gestion de la temporalité dérive de la non -maîtrise des valeurs aspectuelles :

- Soudain, il voyait un petit garçon qui est assis à l'angle de cette grotte et pleure. Ce garçon porte des vêtements qu'il y a des sangs(C.4).

- tous ce qu'ils pêche à sa maison. Soudain, le pêcheur était très peur pour -ce nuit car les voleurs vont voler ses poissons (Copie 3).

- soudain, sortait une belle sirène. Elle s'appele Lina, cette sirène est comme une princesse. le pêcheur a peur au même temps il est joyeux par ce que Lina était belle (Copie 6).

- soudin, il voyait quelque chose grand dans la mer quand il pêchait. Soudin, il sortait une sirène dans la profondeur. L'homme il était très peur mes il ne peut pas courir parce que la sirène est attrapée par un bâton magique...(C.7)

- Tout à coup, venait un animal et attaquait le voleur car le pêcheur donne toujours à lui les poissons...(C.8).

- Soudain, il sortait dans la mer un gros poisson et le jeune homme avait peur et se noyait...soudain un homme du village il trouve la gros poisson...(C.9)

- Tout de suite, le jeune homme se réveillait dans une belle palais et trouvait la sirène assise près de lui...(C.43).

- soudain, il trouvait un renard qui pleure. Le pêcheur est stupéfait(C.26)

- Après des mois et des mois, le pêcheur alla dans un jardin à côté de la mer .Elle resta longtemps à côté de lui une belle femme(Copie 6).

- Comme d'habitude il vendut les poissons que va pêcher et il doit porter ce qui reste pour le manger(C.8)

- Soudain le jeune homme sortait vitement parce qu'il vivait à côté de la mer puis il trouve un pygmée prennait une canne ...(C.13)

Il ressort de ce relevé que les lycéens n'arrivent pas à exploiter le temps convenable du récit, à différencier entre les circonstances secondaires et les actions principales. Les déviations commises émanent de la confusion des deux temps principaux de la narration. Rappelons que le texte narratif se base essentiellement sur le couple P.S. -IMP. comme temps fondamentaux permettant aux événements de se dérouler selon leur importance dans la trame narrative. A ce propos, l'imparfait est souvent réservé aux descriptions vu son caractère non borné et sécant en ce sens qu'il présente le procès dans son déroulement sans indication de commencement ni de fin, ce qui le rend particulièrement apte à servir d'arrière - plan, de toile de fond. Ainsi, à l'effet statique des événements exprimés à l'imparfait s'oppose l'effet dynamique de la progression narrative introduite par le P.S.

Dans cette perspective, Weinrich (1973) a fait appel à l'opposition passé simple (P.S.) /imparfait (IMP.) dans le cadre de ce qu'il appelle « la mise en relief». Ainsi, pour lui, le P.S. est le temps du premier plan, l'imparfait introduit quant à lui le second plan (arrière-plan). En évoquant le rôle de ces deux temps en français, l'auteur affirme " celle-ci n'est autre que de donner du relief au récit en l'articulant par une alternance récurrente entre premier plan et arrière plan. L'imparfait est dans le récit le temps de 
l'arrière - plan, le passé simple le temps du premier - plan. » (Weinrich, 1973: 115). Ce dernier indique la succession des actions dans la narration, dont chacune collabore à la progression $\mathrm{du}$ récit. A l'antipode, l'imparfait exprime une action envisagée comme cadre, décor ou circonstance accessoire de la narration, sans que le commencement ou la fin de cette action soient particulièrement pris en compte.

\subsubsection{Substitution du passé composé au plus que parfait}

- il a parti pour pêchait avec le chien qui l'a sauvé. Quand il a arrivé à la mer, il trouve un coffre plein $\mathrm{d}$ 'or.il était complétement surpris (C.8).

- quand il a lancé la canne il ne peut pas la tirer mais la surprise était une boite(C.12)

- quand il a fini, il retourne chez lui pour dire cela à sa femme(C.15).

- Quand l'enfant est sorti, il entre dans une boite(C.11).

- Soudain, dans sa chambre, il trouve une femme qui pleure tout seul, son visage a complétement attiré le pêcheur. Et il l'a questionné de ce qui a provoqué sa tristesse(C.43).

Ces quelques énoncés incohérents révèlent que les apprenants ignorent l'emploi judicieux de l'antériorité (C.12, C.15 C.11), du discours indirect (C.43) intégré maladroitement dans la copie de l'apprenant. Les temps du récit ne sont pas intériorisés par les apprenants malgré la présence effective d'un module intitulé "typologie textuelle» enseigné au début de l'année scolaire pour tous les troncs communs. L'un des types qui attire l'attention est celui du texte narratif. Soulignons que ce type est enseigné dès le primaire mais les problèmes persistent encore dans son appropriation liés principalement à l'absence d'une compétence linguistique basique qui facilitera la transmission du savoir et du savoir-faire. En réalité cette compétence fait défaut dès le primaire, c'est pourquoi l'apprenant sera immotivé puisqu'il ne comprend pas la communication pédagogique en classe. De surcroit, ses besoins langagiers, culturels ne sont pas atteints du moment qu'il ne parle pas ce médium à la maison ou entre les amis. De ce fait, la relation curriculaire, celle d'enseignement et celle d'apprentissage deviendront primordiales pour optimiser toute action socio -didactique. Ces trois relations pourront réaliser des prestations scolaires optimales à condition qu'elles soient utilisées à bon escient.

\subsection{Propositions didactiques}

Nous sommes convaincu que la façon dont sont enseignés les temps verbaux au niveau du secondaire qualifiant, et principalement les temps du récit, doit être revue et améliorée. Du fait que la distance entre la langue arabe et la langue française dans l'expression du temps est importante (par exemple la diversité des temps du passé en français), l'enseignant du français doit insister sur cet aspect du récit, à savoir la temporalité. Il ne suffit pas de demander à un apprenant de conjuguer un verbe au temps qui convient puisque finalement tous les temps peuvent convenir, tout dépend de la valeur qu'on leur donne dans un énoncé. Aussi faut-il lui enseigner quand et comment utiliser tel temps à tel moment du récit.

Il sera judicieux de faire travailler les lycéens surtout du Tronc commun sur des textes où la cible sera la conjugaison, le temps, le mode, l'aspect ainsi que la concordance des temps pour optimiser l'apprentissage de la temporalité. En outre, l'enseignant peut faire appel à la Technologie de l'Information et de Communication pour l'Enseignement (TICE) pour résoudre certaines déficiences du système temporel. Il est de facto fructueux de recourir à la grammaire de texte et l'analyse du discours, en ne se limitant pas, comme la plupart des grammaires et des méthodes de FLE, à la grammaire de phrase

Comme le programme du lycée qualifiant contenant des œuvres intégrales, il est impératif de maîtriser les différentes caractéristiques de la temporalité. Nous ne pourrons pas étudier les autres procédés narratifs (sommaire, ellipse, analepse, 
prolepse, etc.) si nous ne maîtrisons pas les valeurs modales, aspectuelles et temporelles.

\section{CONCLUSION}

Dans la plupart des récits, les élèves ont du mal à démarrer leur narration à l'aide de temps précis, aussi recourent-ils à plusieurs temps en même temps (présent, passé composé, imparfait, passé simple, etc.). Ce n'est que lors de la complication qu'une répartition des temps narratifs commence à se dégager dans quelques copies.

Les stratégies utilisées par les apprenants ont été différentes et ont contribué à comprendre les hypothèses liées au fonctionnement des productions idiosyncrasiques. En plus, il nous est impossible de décrire en détail les problèmes particuliers des tiroirs verbaux dans les composantes individuelles. L'interprétation des textes nécessite des connaissances socioculturelles approfondies. A cet égard, les différentes substitutions produites par nos apprenants suffisent à montrer que les élèves du TCS éprouvent encore des difficultés quant à la maîtrise des règles du mode, de l'aspect et du temps. Cela peut être expliqué par différentes raisons :

- Méconnaissance de la notion du temps: Les lycéens du TCS n'appréhendent pas la tripartition du temps: passé/présent/futur. Par conséquent, ils confondent les emplois du temps. De surcroit, ils méconnaissent la notion d'antériorité $d u$ fait qu'ils enchaînent le tiroir de la subordonnée sur la principale

- Méconnaissance de la notion d'aspect: nos apprenants confondent les notions de la durée, de l'accompli, de l'inaccompli, etc. En plus, ils confondent souvent les temps simples et les temps composés.

- L'interférence: puisque le système verbal de l'arabe est différent de celui du français, les lycéens du TCS font souvent appel au transfert linguistique particulièrement le temps $\mathrm{du}$ verbe pour véhiculer la temporalité.

\section{REMERCIEMENTS}

Je tiens tout particulièrement à remercier Hassan Boujghagh, A. Amsidder, Ennassiri Hassan et Mokhtar El Mohal pour leurs grandes disponibilités et leurs soutiens constants, sur le plan professionnel et personnel. Un grand merci à mon ami, Kennate Mohammed, qui m'a appuyé constamment dans mes travaux.

\section{RÉFÉRENCES}

Al wohaib, M. (2012). Modélisation et formalisation de l'aspect et $d u$ temps verbal arabe et français : implémentation didactique vers le français sur Internet. Thèse en Sciences du langage Université de Franche-Comté. https://tel.archives-ouvertes.fr/tel01331099.

Azzopardi S. et Bres J. (2011). Temps verbal et énonciation. Le conditionnel et le futur en français : l'un est dialogique, l'autre pas (souvent) », Cahiers de praxématique $56, \quad 53-76$

http://journals.openedition.org/ praxematique $/ 1570$.

Benveniste, E. (1966). Les relations de temps dans le verbe français. Problèmes de linguistique générale, Tome 1, pp. 237250. Paris., Gallimard.

Bronckart, J. P. et alii. (1985). Le fonctionnement des discours. Un modèle psychologique et une méthode d'analyse. Neuchâtel : Delachaux et Niestlé.

Charolles M. (1995). Cohésion, cohérence et pertinence $\mathrm{du}$ discours. Travaux de linguistique, 29, 125-151.

Chiss, Jean-Louis et David, Jacques (2011). Linguistique textuelle et didactique du français. Le français aujourd'hui 5, 155166. Repéré à URL : www.cairn.info/revue-le-francaisaujourd-hui-2011-5-page-155.htm.

Favart, M et Chanquoy, L. (2007). Les marques de cohésion comme outils privilégiés de la textualisation: une comparaison entre élèves de CM2 et 
adultes experts. Langue française 155 , 51-68.

Fuchs, C., Le Goffic, P. et Maillard, M. (2015). Emploi des temps et cohérence textuelle dans l'occupation des sols. Revue Sciences/Lettres. URL : http://journals.openedition.org/rsl/794. Halliday M. A. K. et Hasan R. (1976). Cohesion in English, Londres, Longman.

Mahmudova, S. (2017). Les connecteurs, de la grammaire à la littéracie. Lidil, 56, 1-15. URL

http://journals.openedition.org/lidil/47 57 ; DOI : 10.4000/lidil.4757

Tarnaoui M. M. (2019). La cohésion textuelle dans la production écrite des lycéens marocains : déviations anaphoriques et stratégies didactiques. Thèse de doctorant inédite en didactique du FLE. Faculté des Lettres et des Sciences Humaines, Université Ibn Zohr, Agadir.

Vandendorpe, C. (1995). Au-delà de la phrase : la grammaire du texte. Pour un nouvel enseignement de la grammaire. Sous la direction de Suzanne Chartrand, Montréal, Éditions Logiques, 1995, 83-105.

Vigier Denis (2012). Linguistique textuelle et enseignement/apprentissage du FLES. FDM Recherches et applications Vigier Denis (2012). Linguistique textuelle et enseignement/apprentissage du FLES. Le français dans le monde: Recherches et applications, 51, 34-49.

Weinrich, H. (1973). Le temps, trad. fr. de Michèle Lacoste. Paris, Seuil. 